\title{
Acquired Chiari I Malformation Secondary to Spontaneous Intracranial Hypotension Syndrome and Persistent Hypoglycemia: A Case Report
}

\author{
(1) Hasan Önal ${ }^{1}$, (1) Atilla Ersen ${ }^{2}$, (1) Hakan Gemici ${ }^{3}$, (1) Erdal Adal ${ }^{1}$, (1) Serhat Güler ${ }^{4}$, (1) Serdar Sander ${ }^{5}$, (1) Sait Albayram ${ }^{6}$ \\ 1 University of Health Sciences, Kanuni Sultan Süleyman Training and Research Hospital, Clinic of Pediatric Endocrinology and Metabolism, \\ istanbul, Turkey \\ 2 University of Health Sciences, Okmeydanı Training and Research Hospital, Clinic of Pediatrics, Istanbul, Turkey \\ 3 University of Health Sciences, Kanuni Sultan Süleyman Training and Research Hospital, Clinic of Pediatrics, Istanbul, Turkey \\ ${ }^{4}$ Bezmialem Vakıf University Faculty of Medicine, Department of Pediatric Neurology, Istanbul, Turkey \\ 5 University of Health Sciences, Kanuni Sultan Süleyman Training and Research Hospital, Clinic of Pediatric Surgery, Istanbul, Turkey \\ 6istanbul University Cerrahpaşa Faculty of Medicine, Department of Radiology, Istanbul, Turkey
}

\section{What is already known on this topic?}

Spontaneous intracranial hypotension $(\mathrm{SIH})$ is a rare and potentially serious pathological syndrome in childhood. Concomitant presentation of Chiari I malformation with SIH has rarely been reported. Diagnostic criteria wide-ranging due to variable clinical manifestations. Myelography computerized tomography and epidural blood patch are reliable diagnostic and treatment modalities.

\section{What this study adds?}

Chiari I malformation may mimic spontaneous intracranial hypotension $(\mathrm{SIH})$ and to provide ideal therapy requires recognition of $\mathrm{SIH}$. Persistent hypoglycemia was an early central feature of our patient which is an unusual finding in SIH. Some possible causes of hypoglycemia in SIH are discussed and we present vagotomy as a new treatment modality.

\section{Abstract}

Spontaneous intracranial hypotension (SIH) is a rare and potentially serious condition in childhood. Cerebrospinal fluid (CSF) volume depletion is thought to be the main causative feature for intracranial hypotension and results from a spontaneous CSF leak, often at the spine level. SIH is increasingly diagnosed in clinical practice, although it manifests a varied symptomatology. The downward displacement of the brain, sometimes mimicking a Chiari I malformation, has rarely been reported. We present a case of a SIH with Chiari I malformation accompanied by an unusual clinical presentation of persistent hypoglycemia.

Keywords: Intracranial hypotension, hypoglycemia, vagotomy

\section{Introduction}

Spontaneous intracranial hypotension $(\mathrm{SIH})$ is a rare condition with an estimated prevalence of only one in 50.000 individuals (1). The clinical spectrum of SIH is quite variable and includes headache, neck stiffness, cranial nerve dysfunction, radicular arm pain and symptoms of diencephalic or hindbrain herniation $(1,2)$. Intracranial hypotension is a well-recognized sequel of a spontaneous cerebrospinal fluid (CSF) leak, particularly in cases in which the leak involves the thoracic spine (3). The cause for these CSF leaks remains unclear, but authors have postulated minor trauma, weakness of the dural sac or a combination of both $(4,5)$. More cases are being diagnosed due to advances in imaging, but the diagnosis is still challenging because of the number of atypical, unconfirmed and doubtful cases. The current diagnostic criteria have a wide spectrum due to very variable clinical manifestations. The diagnosis of
Address for Correspondence: Hasan Önal MD, University of Health Sciences, Kanuni Sultan Süleyman Training and Research Hospital, Clinic of Pediatric Endocrinology and Metabolism, İstanbul, Turkey Phone: +90532 5092988 E-mail: hasanonal@hotmail.com ORCID ID: orcid.org/0000-0001-9676-7086 ${ }^{\circ}$ Copyright 2018 by Turkish Pediatric Endocrinology and Diabetes Society

The Journal of Clinical Research in Pediatric Endocrinology published by Galenos Publishing House.
Conflict of interest: None declared Received: 31.01 .2018 Accepted: 25.02 .2018 
SIH is mainly based on presence of an orthostatic headache together with at least one of the following: low CSF pressure, sustained improvement of symptoms after epidural blood patching, demonstration of an active spinal CSF leak and cranial magnetic resonance imaging (MRI) changes demonstrating intracranial hypotension (6). Myelography computerized tomography is the most reliable method for the accurate localization of the CSF leak (7). In most cases, epidural blood patch is the main treatment modality (8).

Chiari I malformation is defined radiographically as a simple displacement of the cerebellar tonsils $5 \mathrm{~mm}$ or greater below the foramen magnum and is distinguished from Chiari II and Chiari III malformations occurring with myelodysplasia and cervical encephalocele, respectively (9). Spontaneous CSF leakage with development of $\mathrm{SIH}$ and acquired Chiari I malformation due to lumbar spinal CSF diversion procedures have both been well described. However, concomitant presentation of both syndromes has rarely been reported. Not to be confused with idiopathic Chiari I malformation, ideal therapy requires recognition of the syndrome and treatment directed to the site of the spinal CSF leak (10).

This is a case of a 13-year-old girl with acquired Chiari I malformation secondary to SIH with a unique coexistence of persistent hypoglycemia.

\section{Case Report}

A 13-year-old girl was admitted to the pediatric emergency unit of Kanuni Sultan Süleyman Training and Research Hospital suffering from hypoglycemia, syncope and convulsive seizures. She had no notable health problem in her past medical history up to 11.5 years of age. Thereafter, she had six subsequent hospital admissions in the previous 1.5 years, mostly at emergency services for hypoglycemic convulsions and syncope attacks. She was born at term, weighing $3100 \mathrm{~g}$ from non-consanguineous parents after an uncomplicated delivery. On evaluation of her records, it was found that she had undergone hypoglycemic periods 2-3 times per day, but syncope attacks were independent from hypoglycemic episodes. She was diagnosed with hyperinsulinemia with a serum glucose level of $29 \mathrm{mg} / \mathrm{dL}$ with a concomitant serum insulin level of 25 IU/L. Positron emission tomography and abdominal ultrasonography were performed to determine the etiology of hyperinsulinemia, but revealed normal anatomic findings. At neurological counseling electroencephalography showed bilateral delta waves with spikes and cranial MRI revealed a 7 $\mathrm{mm}$ herniation of the cerebellar tonsils from the foramen magnum (Figure 1). Further work-up with brainstem auditory evoked potentials and somatosensory evoked potentials, cardiac evaluation with echocardiography and holter monitoring revealed normal findings. Hypoglycemic episodes resolved in the following weeks but, although reduced in number, syncope episodes persisted. Pediatric psychiatry counseling results during her previous admission were not contributory.

At her present admission, the patient's body weight was 55 $\mathrm{kg}$ (90th percentile) and height was $145 \mathrm{~cm}$ (25th percentile). Laboratory investigations revealed hypoglycemia (serum glucose level: $30 \mathrm{mg} / \mathrm{dL}$ ) with a high insulin level of $50 \mathrm{IU} / \mathrm{L}$. Serum C-peptide level was $5 \mathrm{pmol} / \mathrm{mL}$ (N: 0.5-1.30 pmol/mL) and cortisol $23 \mu \mathrm{g} / \mathrm{dL}$ (N: 6.2-19.4 $\mathrm{gg} / \mathrm{dL}$ ). After intravenous glucose, intramuscular glucagon and methylprednisolone treatment, glucagon infusion was initiated. Glucose levels were 40 to $65 \mathrm{mg} / \mathrm{dL}$ during the first 24 hours, but surprisingly, glucose levels of $80-90 \mathrm{mg} / \mathrm{dL}$ were detected in the course of 24 hours after stopping the infusion. Oral glucose tolerance test (OGTT) showed hypoglycemia at the 30th minute (glucose: $25 \mathrm{mg} / \mathrm{dL}$ ) with an insulin level of $300 \mathrm{IU} / \mathrm{L}$. We monitored our patient's daily glucose levels by continuous glucose monitoring system [CGMS System Gold ${ }^{\circledR}$ (Medtronic Minimed, Northridge, CA)] and 14 hypoglycemia episodes

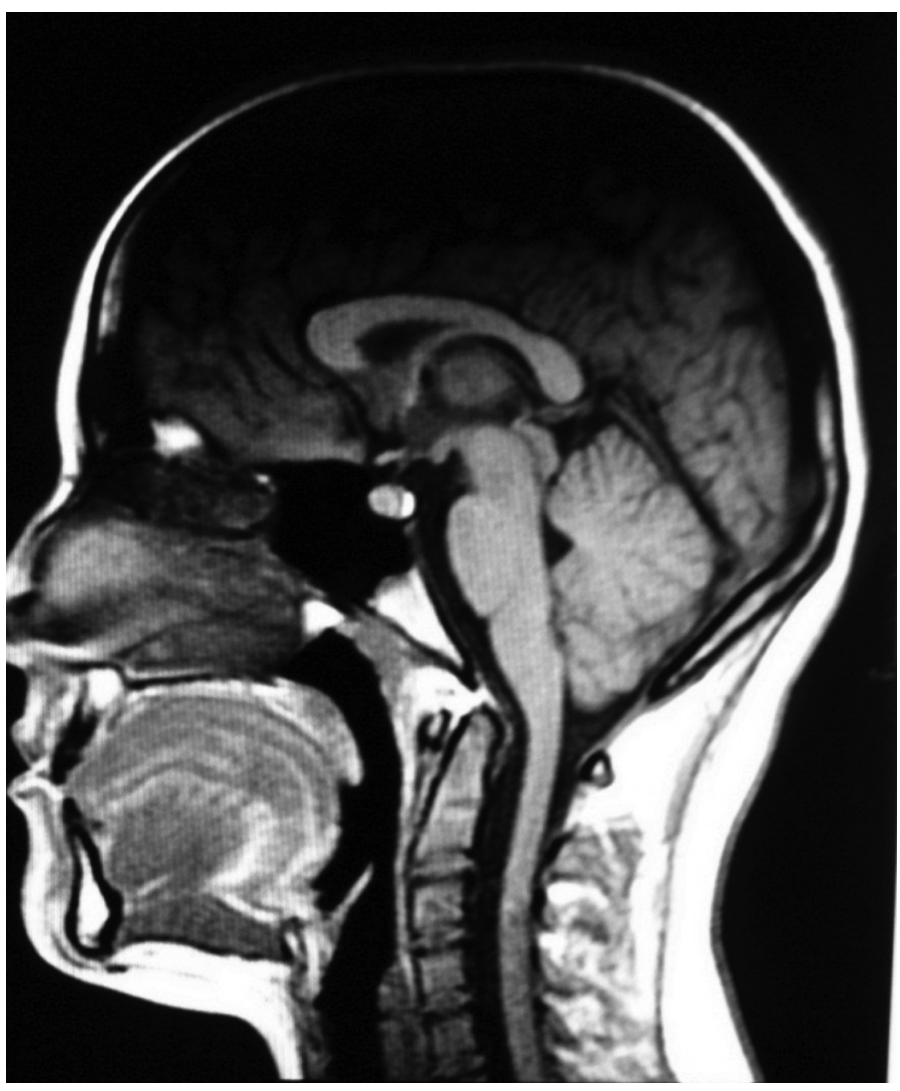

Figure 1. A simple displacement of the cerebellar tonsils 7 $\mathrm{mm}$ below the foramen magnum compatible with Chiari I malformation 
were noted, most occurring during sleep or defecation or in the postprandial period with a maximum glucose level of $70 \mathrm{mg} / \mathrm{dL}$ in three days of follow-up. We measured glucose levels before and after defecation; results were $85 \mathrm{mg} / \mathrm{dL}$ and $29 \mathrm{mg} / \mathrm{dL}$ respectively. No adrenergic symptoms were observed during hypoglycemic episodes. Diazoxide (40 $\mathrm{mg} / \mathrm{kg})$ and octreotide $(40 \mu \mathrm{g} / \mathrm{kg})$ treatment had no effect. Dysarthria was noted in the first month of hospitalization with frequent hypoglycemia episodes. During this period, syncope attacks were observed four times, independent of hypoglycemia. Additionally, the patient had severe biparietal headache episodes in the morning lasting for two hours. These were unrelated to hypoglycemia and were followed by anisocoria. Myelography was performed for SIH and verified with two CSF leaks originating at the lumbar 2 level. The patient underwent a procedure of autologous epidural blood patch at the CSF leak site (Figure 2), with good clinical results including complete control of her episodes of syncope, headache and hypoglycemia.

However, hypoglycemia recurred with dysarthria after two months and was attributed to displacement of the cerebellar tonsils, due to an epidural patch failure. Although the patient remains in good clinical condition after two subsequent epidural patch surgery interventions, neurologic problems and hypoglycemia persisted. Truncal vagotomy and partial pancreatectomy were planned for persistent hypoglycemia, because glucose levels were continuously under $29 \mathrm{mg} / \mathrm{dL}$. At the initiation of this surgical intervention serum glucose was $25 \mathrm{mg} / \mathrm{dL}$. Glucose level spontaneously normalized and was about $100 \mathrm{mg} / \mathrm{dL}$ during anesthesia. Truncal vagotomy was performed firstly. In order to test the efficiency of vagotomy, dextrose $10 \%$ solution infusion was initiated instead of $0.9 \%$ sodium chloride and serum glucose level increased to $180 \mathrm{mg} / \mathrm{dL}$. Distal, partial pancreatectomy was performed additionally, to prevent another surgery risk.


Figure 2. Two cerebrospinal fluid leaks at the lumbar 2 level and the procedure of autologous epidural blood patch
Post operatively diabetes mellitus developed. The patient was discharged with single-dose insulin glargine treatment and her follow-up has been successful for four years.

\section{Discussion}

In clinical practice, SIH may manifest itself as a loss of the prepontine cistern due to leak of CSF, with flattening of the brainstem and downward herniation of the cerebellar tonsils, which may mistakenly lead to a diagnosis of Chiari I malformation $(10,11)$. Our patient was diagnosed initially as a Chiari I malformation, but thereafter, with evaluation of symptoms and laboratory results, a diagnosis of SIH was reached.

SIH is initially suspected on the basis of presenting signs and symptoms such as headache, syncope and some neurological problems. However, in our patient, hypoglycemia was the leading clinical feature and this symptom is not a usual finding in SIH patients.

It has been suggested that hyperinsulinism is responsible for the hypoglycemia. Rekate et al (12) reported four cases diagnosed with Chiari malformation who were suffering from intermittent hyperinsulinemic hypoglycemia and proposed that vagal hypertonia, caused by variation in intracranial pressure, affected the pancreas leading to hypoglycemia in their patients. Tarani et al (13) proposed that the brainstem compression due to hindbrain herniation leads to dysfunction of the normal homeostatic mechanisms to correct hypoglycemia and direct stimulation of the vagal nuclei stimulates pancreatic islet cells to secrete insulin. In our patient, hypoglycemia mostly occurred during parasympathetic activities such as when in a postprandial state, defecation or sleep. Moreover, adrenergic activities had never been observed even with severe hypoglycemic episodes, thus this condition may be attributed to autonomic failure related to parasympathetic dominancy due to vagal stimulus. Vagal efferent activity starts with stimulus of oropharyngeal receptors by oral intake and increases with gastrointestinal peristaltic activity, consequently leading to insulin release, inhibition of norepinephrine from splanchnic nerves, gluconeogenesis and activation of glycogen synthesis (14). Vagal stimulus also produces an early phase of insulin response with postprandial insulin release $(15,16)$. The occurrence of hypoglycemia at the $30^{\text {th }}$ minute of the OGTT with a high insulin level is noteworthy and supports a vagal effect. Moreover, in our opinion the syncope episode in our patient may be related to unbalanced reflexes of the sympathetic system (17).

SIH in childhood is rare (18). Occurrence of orthostatic headaches in the morning suggested a diagnosis of $\mathrm{SIH}$ 
and the myelogram showed two dural puncture areas in the lumbar region (Figure 2). Treatment with the patch procedure improved hypoglycemia and other symptoms with resolving caudal displacement of the cerebellar tonsils. Rekate et al (12) used continuous-drip feeding for hypoglycemia in their cases with Arnold Chiari syndrome. Unfortunately, persistent hypoglycemia relapsed after epidural patch procedure failures in our patient. In concordance with our estimation of parasympathetic dominancy, truncal vagotomy and partial pancreatectomy were planned as a radical therapy for her persistent hypoglycemia. Surgery was started with a serum glucose level of $25 \mathrm{mg} / \mathrm{dL}$, and surprisingly, serum glucose level normalized with anesthesia induction. This outcome may be related to a possible effect of anesthesia on sympathetic-parasympathetic balance (19). Additionally, glucose response after the vagotomy procedure during surgery verified the role of a vagal effect on hypoglycemia. Diabetes mellitus presented after the surgery due to partial pancreatectomy and our patient continues to take singledose glargine insulin treatment.

In clinical practice, intracranial hypotension syndrome may manifest with a variety of symptoms, one of which may be persistent hypoglycemia. This paper reports the findings in a patient with this syndrome, along with some pathophysiological considerations, diagnostic processes and possible treatment modalities in SIH patients with persistent hypoglycemia.

\section{Ethics}

Informed Consent: A written informed consent was obtained from the parent.

Peer-review: Externally peer-reviewed.

\section{Authorship Contributions}

Surgical and Medical Practices: Hasan Önal, Concept: Erdal Adal, Design: Zerrin Önal, Data Collection or Processing: Serdar Sander, Analysis or Interpretation: Sait Albayram, Literature Search: Atilla Ersen, Hakan Gemici, Writing: Atilla Ersen.

Financial Disclosure: There is no financial or other relationship.

\section{References}

1. Schievink WI. Spontaneous spinal cerebrospinal fluid leaks: a review. Neurosurg Focus 2000;15;9:8.
2. Chung SJ, Kim JS, Lee MC. Syndrome of cerebral spinal fluid hypovolemia: clinical and imaging features and outcome. Neurology 2000;55:1321-1327.

3. Mokri B. Spontaneous intracranial hypotension. Curr Pain Headache Rep 2001;5:284-291.

4. Ferrante E, Citterio A, Savino A, Santalucia P. Postural headache in patient with Marfan's syndrome. Cephalalgia 2003;23:552-555.

5. Pannullo SC, Reich JB, Krol G, Deck MD, Posner JB. MRI changes in intracranial hypotension. Neurology 1993;43:919-926.

6. Schievink WI, Dodick DW, Mokri B, Silberstein S, Bousser MG, Goadsby PJ. Diagnostic criteria for headache due to spontaneous intracranial hypotension: a perspective. Headache 2011;51:1442-1444. Epub 2011 Jun 9

7. Limaye K, Samant R, Lee RW. Spontaneous intracranial hypotension: diagnosis to management. Acta Neurol Belg 2016;116:119-125. Epub 2015 Dec 10

8. Mokri B. Spontaneous Intracranial Hypotension. Continuum (Minneap Minn) 2015;21:1086-1108.

9. Elster AD, Chen MY. Chiari I malformations: clinical and radiologic reappraisal. Radiology 1992;183:347-353.

10. Atkinson JL, Weinshenker BG, Miller GM, Piepgras DG, Mokri B. Acquired Chiari I malformation secondary to spontaneous spinal cerebrospinal fluid leakage and chronic intracranial hypotension syndrome in seven cases. J Neurosurg 1998;88:237-242.

11. Mokri B, Hunter SF, Atkinson JL, Piepgras DG. Orthostatic headaches caused by CSF leak but with normal CSF pressures. Neurology 1998;51:786-790

12. Rekate HL, Nadkarni TD, Teaford PA, Wallace D. Brainstem dysfunction in Chiari malformation presenting as profound hypoglycemia: presentation of four cases, review of the literature, and conjecture as to mechanism. Neurosurgery 1999;45:386-391.

13. Tarani L, Del Balzo F, Costantino F, Properzi E, D’Eufemia P, Liberati N, Spalice A. Chiari type I malformation, syncope, headache, hypoglycemia and hepatic steatosis in an 8-year old girl: a causal association? Pediatr Rep 2010;2:8

14. Brightbill TC, Goodwin RS, Ford RG. Magnetic resonance imaging of intracranial hypotension syndrome with pathophysiological correlation. Headache 2000;40:292-299.

15. Teff KL, Levin BE, Engelman K. Oral sensory stimulation in men: effects on insulin, C-peptide, and catecholamines. Am J Physiol 1993:265:1223-1230

16. Shimazu T. Regulation of glycogen metabolism in liver by the autonomic nervous system $\mathrm{V}$. activation of glycogen synthetase by vagal stimulation. Biochim Biophys Acta 1971;252:28-38.

17. Ahren B, Taborsky GJ Jr, Porte D Jr. Neuropeptidergic versus cholinergic and adrenergic regulation of islet hormone secretion. Diabetologia 1986;29:827-836

18. Schievink WI, Maya MM, Louy C, Moser FG, Sloninsky L. Spontaneous intracranial hypotension in childhood and adolescence. J Pediatr 2013;163:504-510. Epub 2013 Feb 28

19. Latson TW, McCarroll SM, Mirhej MA, Hyndman VA, Whitten CW, Lipton JM. Effects of three anesthetic induction techniques on heart rate variability. J Clin Anesth 1992;4:265-276. 